\title{
Deflection of Thin Circular Elastic Plates Under Symmetrically Distributed Loading
}

\author{
A. F. Kirstein and R. M. Woolley \\ Institute for Basic Standards, National Bureau of Standards, Washington D.C. 20234 \\ (November 16, 1967)
}

\begin{abstract}
A special application of Bassali's solution for transverse flexure of thin elastic plates supported at several points is presented for the case of concentric symmetrically distributed load. Equations for deflection are presented for the case where the point supports lie within the loaded area. Under special conditions the numerical results from these equations coincide with those of Nadai's.

Key Words: Bassali's theory, deflection, elasticity, flexure, point supports, symmetrically distributed load, thin plates.
\end{abstract}

\section{Introduction}

Previous work $[1]^{1}$ dealt with the deflection of centrally loaded thin circular elastic plates on equally spaced point supports in which the supports were located along a concentric circle outside of the central loaded area. A sequel to this previous work is presented in this paper where the case in which the point supports lie within the loaded area is considered.

Theoretical work given by Bassali [2] is specialized to develop expressions for deflection which consider the symmetrically distributed load in general and the uniformly distributed load in particular.

Previous experimental results $[1,3]$ have been shown to be in good agreement with the results of other specialized treatments of Bassali's theory for plates having thicknesses to span radii ratios up to 0.08 with span radii to plate radii ratios as small as 0.63 . Even though no experimental results are presented in this paper, no lesser agreement between experimental and theoretical results should be expected for the case considered herein.

\section{List of Symbols}

$x, y$ rectangular Cartesian coordinates

$r=\sqrt{x^{2}+y^{2}}$, radial polar coordinate

$\theta$ arctan $(y / x)$, polar coordinate angle

$c$ radius of the plate

$b$ radius of transversely loaded area of plate (Region 1)

$a$ span radius, of circle on which supports lie

$h$ thickness of the plate

'Figures in brackets indicate the literature references at the end of this paper. 


$$
\begin{aligned}
& m \text { number of support points }(m \geqslant 3) \\
& \alpha=2 \pi / \mathrm{m}, \text { polar angle between support points } \\
& \theta_{s}=s \alpha, \text { polar angle subtended by the sth support point }(s=1,2, \ldots, m) \\
& \varphi_{s}=\theta-\theta_{s} \\
& p_{1}=p_{0} r^{n-2}, \text { transverse load intensity over Region } 1 \\
& p_{2}=0, \text { transverse load intensity over Region } 2 \\
& P_{0}=2 \pi p_{0} b^{n} / n, \text { total load on the plate } \\
& b^{\prime}=b \sqrt{n /(n+2)} \\
& \rho=r / c \\
& t=a / c \\
& q=b / c \\
& q^{\prime}=b^{\prime} / c \\
& r_{s}^{\prime}=c^{2} / a \\
& \nu \text { Poisson's ratio } \\
& \kappa=(3+\nu) /(\nu-1) \\
& E \text { modulus of elasticity } \\
& D=E h^{3} / 12\left(1-\nu^{2}\right), \text { the flexural rigidity } \\
& w_{1} \text { deflection of the plate within Region } 1 \\
& w_{2} \text { deflection of the plate within Region } 2 \\
& w_{c} \text { deflection at the center of the plate. }
\end{aligned}
$$

\section{Deflection of the Circular Plate}

The theoretical expressions for the deflection of a plate were obtained from Bassali's work [2] and a previous specialization [1] of his work which dealt with the deflection of centrally loaded thin circular elastic plates on equally spaced point supports. The previous case considered that the point supports were located along a concentric circle outside of the central loaded area (in Region 2). For the case presented here the point supports lie within the loaded area (Region 1). Figure 1 shows the concentric arrangement of the circular plate and defines Regions 1 and 2.

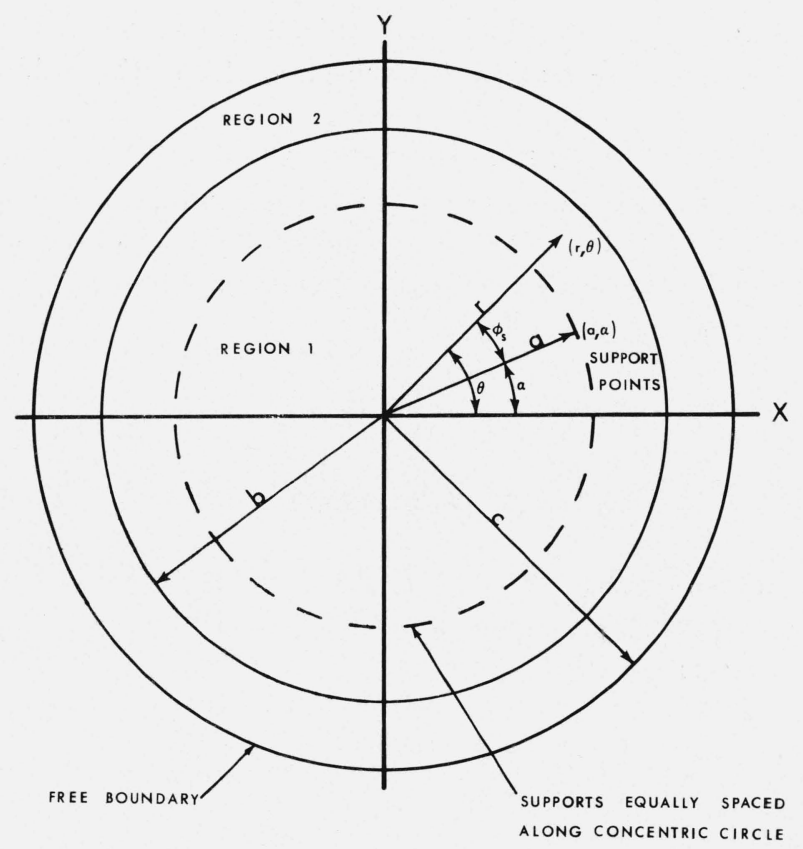

FIGURE 1. Concentric arrangement of the circular plate. 
Previously developed $[1,2]$ general expressions pertaining to symmetrical bending are presented here as eqs (1) to (7). For the case considered here, the development follows the same general reasoning. The deflections of the plate in Regions 1 and 2 are represented by

$$
w_{1}=w_{0}+\frac{P_{0}}{8 \pi D}\left[\left(r^{2}+b^{\prime 2}\right) \ln q-\frac{1}{m} \sum_{s=1}^{m} R_{s}^{2} \ln \frac{R_{s}}{c}+\left(1-\frac{1}{n}\right)\left(r^{2}-b^{2}\right)+\frac{4\left(r^{n+2}-b^{n+2}\right)}{n(n+2)^{2} b^{n}}\right]
$$

where $n$ is the load distribution parameter, and

$$
w_{2}=w_{0}+\frac{P_{0}}{8 \pi D}\left[\left(r^{2}+b^{\prime 2}\right) \ln \rho-\frac{1}{m} \sum_{s=1}^{m} R_{s}^{2} \ln \frac{R_{s}}{c}\right],
$$

respectively, where

$w_{0}=\frac{P_{0}}{8 \pi m \kappa D}\left[\sum_{s=1}^{m} R_{s}^{2} \ln \frac{R_{s}^{\prime}}{r_{s}^{\prime}}+\left(\kappa^{2}-1\right) c^{2} \operatorname{Re}\left\{L_{m}(\zeta)\right\}+\frac{m\left(1-\rho^{2}\right)\left(a^{2}+\kappa b^{\prime 2}\right)}{\kappa+1}\right]+\gamma_{1} x+\gamma_{2} y+\gamma_{3}$,

and

$$
\begin{aligned}
R_{s}^{2}(\rho, \theta) & =a^{2}\left(1+\frac{\rho^{2}}{t^{2}}-2 \frac{\rho}{t} \cos \varphi_{s}\right) \\
\frac{R_{s}^{2}}{c^{2}} & =\rho^{2}-2 \rho t \cos \varphi_{s}+t^{2} \\
\frac{R_{s}^{\prime 2}}{r_{s}^{\prime 2}} & =1-2 \rho t \cos \varphi_{s}+\rho^{2} t^{2}
\end{aligned}
$$

$\operatorname{Re}\left\{L_{m}(\zeta)\right\}=-\sum_{n=1}^{\infty}\left[\frac{(\rho t)^{n}}{n^{2}} \sum_{s=1}^{m} \cos n \varphi_{s}\right]+\frac{1}{2} \sum_{s=1}^{m}\left(1-\rho t \cos \varphi_{s}\right) \ln \left(1-2 \rho t \cos \varphi_{s}+\rho^{2} t^{2}\right)$

$$
-\rho t \sum_{s=1}^{m} \sin \varphi_{s} \arctan \frac{\rho t \sin \varphi_{s}}{1-\rho t \cos \varphi_{s}} .
$$

For the case in which the point supports lie within the loaded area (Region 1 ), i.e., $t \leqslant q$, the deflection, $w_{1}$ is taken to vanish at a point support, $z_{k}$. By setting eq (1) equal to zero, $r=a$, and $\rho=t$ an expression for $w_{0}$ is found to be

$$
\begin{aligned}
w_{0}=-\frac{P_{0}}{8 \pi D}\left[\left(a^{2}+b^{\prime 2}\right) \ln q-\frac{1}{m} \sum_{s=1}^{m} R_{s}^{2}\left(a, \theta_{k}\right) \ln \frac{R_{s}}{c}\left(a, \theta_{k}\right)\right. & \\
& \left.+\left(1-\frac{1}{n}\right)\left(a^{2}-b^{2}\right)+\frac{4\left(a^{n+2}-b^{n+2}\right)}{n(n+2)^{2} b^{n}}\right] .
\end{aligned}
$$

Noting that the central plane of the undeformed plate shown in figure 1 is taken to lie in the $x-y$ plane, i.e., $\gamma_{1}=\gamma_{2}=0$, eqs $(3,4,5,6$, and 8$)$ may be combined to give

$$
\begin{aligned}
\gamma_{3}=\frac{P_{0}}{8 \pi \kappa D}\left[a^{2}\left\{A_{m}(t)+\left(1-\kappa^{2}\right) B_{m}(t)\right\}-\kappa\left(a^{2}+b^{\prime 2}\right)\right. & \ln q+\kappa\left(1-\frac{1}{n}\right)\left(b^{2}-a^{2}\right) \\
& \left.+\frac{4 \kappa\left(b^{n+2}-a^{n+2}\right)}{n(n+2)^{2} b^{n}}-\frac{\left(1-t^{2}\right)\left(a^{2}+\kappa b^{\prime 2}\right)}{\kappa+1}\right]
\end{aligned}
$$

${ }^{2}$ Bassali's expression for $\gamma_{3}$ is incorrect in that it contains an additional term, $+2 a^{2} \kappa \ln t$, which does not appear in eq (9). 
where

$$
m A_{m}(t)=\sum_{s=1}^{m}(1-\cos s \alpha)\left[\kappa \ln 2 t^{2}(1-\cos s \alpha)-\ln \left(1-2 t^{2} \cos s \alpha+t^{4}\right)\right],
$$

and

$$
\begin{aligned}
m t^{2} B_{m}(t)=\frac{1}{2} \sum_{s=1}^{m}\left(1-t^{2} \cos s \alpha\right) \ln \left(1-2 t^{2} \cos s \alpha+t^{4}\right) & -\frac{1}{m} \varphi\left(t^{2 m}\right) \\
& -t^{2} \sum_{s=1}^{m} \sin s \alpha \arctan \frac{t^{2} \sin s \alpha}{1-t^{2} \cos s \alpha}
\end{aligned}
$$

where $\varphi(z)$ is defined as $-\int_{0}^{z} \ln (1-\tau) \frac{d \tau}{\tau},|z|<1$.

Under the given conditions of support it is now possible to determine the deflection of the central plane of a thin circular elastic plate when subjected to a concentric symmetrically distributed load. The intensity of the distributed load is $p=p_{1}=p_{0} r^{n-2}$ when $n \geqslant 2$ for Region 1 and $p=p_{2}=0$ for Region 2 ( $n=2$ produces uniform load). The total load on the plate is $P_{0}=2 \pi p_{0} b^{n} / n$.

\section{Uniformly Loaded Plate}

The solution for deflection of the uniformly loaded plate is implicit in the more general equations presented in the preceding section. Since there is some practical interest in the uniformly loaded plate on equally spaced point supports, this section presents the development of the equations.

In this case $b=c, q=1, n=2$, and $b^{\prime 2}=c^{2} / 2$. The appropriate deflection is $w_{1}$, and considering the above equalities eqs ( 1,3 , and 9 ) may be written as

$$
\begin{aligned}
& w_{1}=w_{0}+\frac{P_{0}}{8 \pi \kappa D}\left[-\frac{\kappa}{m} \sum_{s=1}^{m} R_{s}^{2} \ln \frac{R_{s}}{c}+\frac{\kappa\left(r^{2}-c^{2}\right)}{2}+\frac{\kappa\left(r^{4}-c^{4}\right)}{8 c^{2}}\right], \\
& w_{0}=\frac{P_{0}}{8 \pi m \kappa D}\left[\sum_{s=1}^{m} R_{s}^{2} \ln \frac{R_{s}^{\prime}}{r_{s}^{\prime}}+\left(\kappa^{2}-1\right) c^{2} \operatorname{Re}\left\{L_{m}(\zeta)\right\}+\frac{m\left(1-\rho^{2}\right)\left(a^{2}+\frac{\kappa c^{2}}{2}\right)}{\kappa+1}\right]+\gamma_{3},
\end{aligned}
$$

and

$$
\gamma_{3}=\frac{P_{0}}{8 \pi \kappa D}\left[a^{2}\left\{A_{m}(t)+\left(1-\kappa^{2}\right) B_{m}(t)\right\}+\frac{\kappa\left(c^{2}-a^{2}\right)}{2}+\frac{\kappa\left(c^{4}-a^{4}\right)}{8 c^{2}}-\frac{\left(1-t^{2}\right)\left(a^{2}+\frac{\kappa c^{2}}{2}\right)}{\kappa+1}\right] .
$$

Combining eqs $(12,13$, and 14) the deflection anywhere on the central plane of the plate is $w_{1}=\frac{P_{0}}{8 \pi \kappa D}\left[\frac{1}{m} \sum_{s=1}^{m} R_{s}^{2} \ln \frac{R_{s}^{\prime}}{r_{s}^{\prime}}-\frac{\kappa}{m} \sum_{s=1}^{m} R_{s}^{2} \ln \frac{R_{s}}{c}+\frac{\left(\kappa^{2}-1\right) c^{2}}{m} \operatorname{Re}\left\{L_{m}(\zeta)\right\}\right.$

$$
\left.+a^{2}\left\{A_{m}(t)+\left(1-\kappa^{2}\right) B_{m}(t)\right\}+\frac{\kappa\left(r^{2}-a^{2}\right)}{2}+\frac{\kappa\left(r^{4}-a^{4}\right)}{8 c^{2}}+\frac{\left(t^{2}-\rho^{2}\right)\left(a^{2}+\frac{\kappa c^{2}}{2}\right)}{\kappa+1}\right] .
$$


Combining this and eqs $(4,5$, and 6$)$ gives

$$
\begin{aligned}
w_{1} & =\frac{P_{0} a^{2}}{8 \pi \kappa D}\left[\frac{1}{2 m t^{2}} \sum_{s=1}^{m}\left(\rho^{2}-2 \rho t \cos \varphi_{s}+t^{2}\right) \ln \left(1-2 \rho t \cos \varphi_{s}+\rho^{2} t^{2}\right)\right. \\
& -\frac{\kappa}{2 m t^{2}} \sum_{s=1}^{m}\left(\rho^{2}-2 \rho t \cos \varphi_{s}+t^{2}\right) \ln \left(\rho^{2}-2 \rho t \cos \varphi_{s}+t^{2}\right)+\frac{\left(\kappa^{2}-1\right)}{m t^{2}} \operatorname{Re}\left\{L_{m}(\zeta)\right\}+A_{m}(t) \\
& \left.+\left(1-\kappa^{2}\right) B_{m}(t)+\frac{\kappa}{2 t^{2}}\left(\rho^{2}-t^{2}\right)+\frac{\kappa}{8 t^{2}}\left(\rho^{4}-t^{4}\right)-\frac{\left(\rho^{2}-t^{2}\right)\left(1+\frac{\kappa}{2 t^{2}}\right)}{\kappa+1}\right]
\end{aligned}
$$

where $\operatorname{Re}\left\{L_{m}(\zeta)\right\}, A_{m}(t)$, and $B_{m}(t)$ may be determined from eqs (7, 10, and 11), respectively.

The deflection at the center of the plate when the point supports are equally spaced along the periphery may be determined by inserting $\rho=0, t=1$, and $\operatorname{Re}\left\{L_{m}(0)\right\}=0$ into eq (16). The result is

$$
w_{c}=\frac{P_{0} a^{2}}{8 \pi \kappa D}\left[A_{m}(1)+\left(1-\kappa^{2}\right) B_{m}(1)-\frac{5 \kappa}{8}+\frac{\left(1+\frac{\kappa}{2}\right)}{\kappa+1}\right] .
$$

Likewise the deflection at the periphery midway between supports may be determined from eq (16) by setting $\rho=t=1$, yielding

$w_{d}=\frac{P_{0} a^{2}}{8 \pi \kappa D}\left[A_{m}(1)+\left(1-\kappa^{2}\right) B_{m}(1)+\frac{\kappa^{2}-1}{m} \operatorname{Re}\left\{L_{m}(\zeta)\right\}\right.$

$$
\left.+\frac{1-\kappa}{m} \sum_{s=1}^{m}\left(1-\cos \varphi_{s}\right) \ln 2\left(1-\cos \varphi_{s}\right)\right]
$$

These cases represented by eqs (17 and 18) were considered by Nadai [4]. For comparison set $m=3$ and $\nu=\frac{1}{4}$ and we have

$$
w_{c}=0.407 \frac{P_{0} a^{2}}{E h^{3}}
$$

and

$$
w_{d}=0.307 \frac{P_{0} a^{2}}{E h^{3}}
$$

which coincide with the values obtained from Nadai's work.

\section{Summary}

Theoretical expressions are presented to make it possible to determine the deflection of the central plane of a thin circular elastic plate which is subjected to a concentric symmetrically distributed load when the point supports are equally spaced along a concentric circle and lie within the loaded area (Region 1). 
The uniformly loaded plate is treated as a separate case, and the appropriate equations for deflection are presented. The computed deflection at the center of a uniformly loaded plate on point supports equally spaced along the periphery coincides with the results from Nadai's work.

\section{References}

[1] Kirstein, A. F., Pell, W. H., Woolley, R. M., and Davis, L. J., Deflection of centrally loaded thin circular elastic plates on equally spaced point supports, J. Res. NBS 70C (Engr. and Instr.), No. 4, 227-244 (Oct.-Dec. 1966).

[2] Bassali, W. A., The transverse flexure of thin elastic plates supported at several points, Proceedings Cambridge Philosophical Society, 53, 728-743 (1957).

[3] Kirstein, A. F., and Woolley, R. M., Symmetrical bending of thin circular elastic plates on equally spaced point supports, J. Res. NBS $71 C$ (Engr. and Instr.), No. 1, 1-10 (Jan.-Mar. 1967).

[4] Nadai, A., Die Verbiegungen in einzelnen Punkten unterstützter kreisförmiger Platten, Physik. Z. 23, 366-376 (1922).

(Paper 72C1-265) 